\title{
Psychedelic Science of Spirituality and Religion: \\ An Attachment-Informed Agenda Proposal
}

Aaron D. Cherniak ${ }^{1}$, Joel Gruneau-Brulin ${ }^{1}$, Mario Mikulincer ${ }^{2}$, Sebastian Östlind ${ }^{1}$, Robin Carhart-Harris $^{3,4}$, \& Pehr Granqvist ${ }^{1}$

\author{
${ }^{1}$ Stockholm University \\ ${ }^{2}$ Reichman University (IDC Herzliya) \\ ${ }^{3}$ Imperial College London \\ ${ }^{4}$ University of California, San Francisco, USA
}

\begin{abstract}
Author Note
Aaron D. Cherniak https://orcid.org/0000-0002-9114-4290

$*=$ Contributed equally to this work.

The authors have no conflicts of interest to disclose.

Cherniak's work was supported by the 2021 SRF Source Award from the Source Research

Correspondence regarding this article should be addressed to Aaron D. Cherniak (aaroncherniak@gmail.com) or Joel Gruneau-Brulin (joel.gruneau.brulin@psychology.su.se).
\end{abstract} Foundation. 


\begin{abstract}
In this paper, we set an agenda for a psychedelic science of spirituality and religion, based on a synthesis of attachment theory with the Relaxed Beliefs Under pSychedelics (REBUS) model. Attachment theory proposes that people develop internal working models (IWMs) of interactions with others from their relational experiences with caregivers. Such IWMs then function as highlevel priors enabling people, for better and for worse, to predict and organize their interpersonal and religious/spiritual relationships. One mechanism by which efficacious psychedelic interventions may work is by relaxing the grip of rigid, defensive priors (e.g., insecure IWMs with regard to others and God), further amplified by corrective relational experiences with the therapist, God, or others. We outline three key proposals to steer future research. First, individual differences in attachment security predict the phenomenology and integration of psychedelic experiences. Second, efficacious psychedelic therapy facilitates increased attachment security as a clinically relevant outcome. Third, attachment-related dynamics (e.g., a sense of connection to others and God, alleviation of attachment-related worries and defenses) are process-level mechanisms involved in the clinical utility of psychedelic treatment. Finally, we discuss the role of religion and spirituality in psychedelic experiences from an attachment perspective. Keywords: psychedelics; attachment; spirituality; religion; predictive coding
\end{abstract}




\section{ATTACHMENT \& PSYCHEDELIC MYSTICAL EXPERIENCE}

Classical psychedelics, such as psilocybin, lysergic acid diethylamide (LSD), ayahuasca/ $\mathrm{N}, \mathrm{N}$-dimethyltryptamine (DMT), and mescaline, have long been used in religious and healing rituals (Bruhn et al., 2002), and there is a growing interest in their therapeutic potential for treating mental problems (e.g., Johnson et al., 2019). Research has provided supportive evidence for the positive clinical outcomes of psychedelic-intake during therapy and emphasized the therapeutic benefits of the transformative (spiritual or mystical) experiences often engendered by psychedelic trips (e.g., Griffiths et al., 2006). However, we still lack a broad, agreed-upon conceptual framework for explaining the underlying therapeutic processes and mechanisms of change. In the present article, we propose leveraging a synthesis of attachment theory (Bowlby, 1982) and the REBUS model ("RElaxed Beliefs Under pSychedelics"; Carhart-Harris \& Friston, 2019) as a conceptual framework for a psychedelic science of spirituality and religion.

We offer three specific proposals based on attachment theory and the attachment-REBUS synthesis. First, attachment security and insecurities act as predictors of psychedelic experiences and their exploration and integration. Second, increased attachment security is a viable clinical outcome of psychedelic therapy. Third, attachment-related dynamics (e.g., a heightened felt connection with others and God, alleviation of attachment-related worries and defenses) are process-level mechanisms that explain some of the clinical utility of psychedelic treatment.

We develop our arguments in four main sections. In the first, we review attachment theory and its perspective on religion and spirituality. Second, we give a brief overview of psychedelic science, addressing brain processes and subjective effects according to the REBUS model. In the third section, we synthesize attachment theory and the REBUS model. Finally, we discuss implications of the attachment-REBUS synthesis for a psychedelic science of spirituality and religion, note outstanding issues, and chart future directions for research and clinical practice. 


\section{Attachment Theory and Spirituality/Religion}

\section{Attachment Theory}

Attachment theory (Bowlby, 1982) posits that an innate psychobiological mechanism (attachment behavioral system) motivates humans to seek proximity to stronger, wiser, and caring others (attachment figures) in times of need. These figures can provide a safe haven when distressed (comfort, support, reassurance) and a secure base, from which one can explore the world and pursue personal goals with the confidence that support will be available when needed. When attachment figures fully accomplish these functions, they instill a sense of attachment security (or felt security) - beliefs that the world is a safe place and that others are helpful when called upon, with positive implications for well-being and mental health (Bowlby, 1988).

According to Bowlby (1973), interactions with attachment figures are internalized as mental representations of self and others (internal working models, IWMs), which aid the person to organize their behavior in subsequent interactions with these figures and predict the outcome of these exchanges. Interactions with attachment figures who are responsive and supportive promote felt security, contribute to positive IWMs of oneself (as worthy of care) and others (as available to care), and facilitate adaptive self-regulation (Mikulincer \& Shaver, 2016). By contrast, interactions with insensitive (e.g., rejecting, role-reversing, or abusive) attachment figures foster attachment-related insecurities, characterized by preoccupations with self-worth and others' intentions and the development of maladaptive self-regulation strategies.

Over time, IWMs are crystalized into attachment orientations - relatively stable patterns of relational cognitions, emotions, and behaviors (Fraley \& Shaver, 2000). According to Brennan et al. (1998), these orientations can be conceptualized and measured along two dimensions: attachment anxiety (worries about one's lovability) and attachment-related avoidance (distrust of 


\section{ATTACHMENT \& PSYCHEDELIC MYSTICAL EXPERIENCE}

others' goodwill and inhibition of relational closeness). Whereas high avoidance or anxiety reflect attachment insecurities, people who score low on these two dimensions tend to hold a solid sense of attachment security. Beyond these two dimensions, attachment researchers have conceptualized and measured what they call attachment disorganization - a breakdown in attachment-related patterns of attention and behavior (i.e., contradictory and confused behavior) that may occur during stressful and potentially traumatic situations (Main \& Solomon, 1990).

Decades of research support the anxiety-buffering and growth-promoting functions of the sense of attachment security and the positive consequences of making felt security contextually salient (what Mikulincer \& Shaver, 2016, called security priming). Whereas secure attachment facilitates effective coping with undesirable physiological and psychological states, attachment insecurities might hamper emotion regulation. Research indicates that anxious attachment reinforces distress up-regulation, negative self-views, and catastrophic beliefs, whereas avoidant attachment leads people to suppress emotions and inhibit direct contact with inner experiences (Mikulincer et al., 2003). Over time, insecure attachment can become a vulnerability factor for emotional and interpersonal problems, with disorganized attachment as a particular risk factor for psychopathology (Stovall-McClough \& Dozier, 2016). By contrast, secure attachment functions as a source of resilience that sustains well-being and mental health (Mikulincer \& Shaver, 2016). Consequently, attachment security at the outset of psychotherapy and increases in felt security during therapy predict favorable clinical outcomes (Levy et al., 2018).

Although early experiences exert a powerful influence on attachment orientations, which are at least moderately stable, these dispositions are somewhat malleable via new relational experiences (Fraley et al., 2011). In fact, IWMs are tolerably accurate reflections of what has actually happened to a person in one or more important relationships, and they are always subject 


\section{ATTACHMENT \& PSYCHEDELIC MYSTICAL EXPERIENCE}

to revision in response to subsequent relational experiences (Bowlby, 1973). For example, insecure IWMs can be revised through corrective attachment-related experiences, such as in psychotherapy (Levy et al., 2018), and possibly through profound experiences variously denoted as religious, spiritual, or mystical (Granqvist, 2020).

\section{Attachment Theory and Spirituality/Religion}

Attachment theory has been extended to people's perceived relationships with "stronger and wiser" spiritual beings, such as God (Granqvist, 2020). Central to this extension is the sense many people have of being in a personal relationship with benevolent spiritual beings. This is especially the case in the major theistic faith traditions that depict God as a source of love, comfort, and support, reflected in both explicit, doctrinal beliefs and implicit, affect-laden religious experience (Paloutzian \& Park, 2013). The religion-as-attachment model explains how believers' attachment relationship with the divine underlies much of their religious beliefs, behaviors, and experiences, as well as of religion's link to health (Granqvist, 2020).

Believers tend to perceive God as a safe haven in times of need, they make explicit requests for protection and support through prayer and rituals, and they appeal to God as a secure base for resilience and prosperity (Davis et al., 2019). Thus, believers functionally experience God as an attachment figure (Cherniak et al., 2021), and research supports the anxiety-buffering and growth-promoting functions of attachment to God (e.g., Granqvist, Mikulincer et al., 2012). Neuroscientific studies have similarly shown that believers experience God as a relational, dialogical partner when engaging in personal prayer (Schjoedt et al., 2009) and that sensed closeness to God may activate reward-related brain networks (Ferguson et al., 2018).

Like in interpersonal relationships, believers might differ in their sense of attachment security with regard to God, which appears to moderate the link between religion/spirituality and 


\section{ATTACHMENT \& PSYCHEDELIC MYSTICAL EXPERIENCE}

mental health (Granqvist, 2020). Secure attachment to God reflects trust in God's support and a coherent God representation insecure attachment reflects doubts about God's love and one's right to it (anxiety) or reluctance to rely upon God (avoidance), and attachment disorganization may take the form of a fearful representation of a terrifying, capricious deity (cf. Otto's [1925] notion of "tremendum"). Sharp et al. (2019) proposed two representational levels of God-related IWMs: 1) doctrinal-cognitive, explicit representations based on formal religious beliefs, and 2) experiential-affective, implicit representations based on spiritual experiences. Representations' valence and in/congruence interact to affect religiosity and well-being (Pirutinsky et al., 2020).

Two developmental pathways have been proposed to describe how attachment to God is rooted in attachment-related interpersonal experiences (Granqvist \& Kirkpatrick, 2016). First, the correspondence pathway depicts how IWMs from human-human relationships generalize to IWMs of God. Secure IWMs foster benevolent and coherent God representations, whereas insecure IWMs foster malevolent and incoherent God representations. Second, the compensation pathway depicts how God may be used by attachment-insecure people as a surrogate figure to compensate for unsatisfactory attachment human-human relationships.

The correspondence and compensation pathways are tied to different religious/spiritual change profiles. Attachment-secure people's religious changes security are generally gradual and associated with security-related themes (e.g., optimism; Kimball et al., 2013) and theological exploration (Greenwald et al., 2021). Conversely, insecure attachment precipitates more sudden, intense, and emotionally turbulent religious/spiritual changes (Granqvist \& Kirkpatrick, 2004).

Notably, attachment disorganization has been linked with a propensity to enter dissociative mental states (Hesse \& van Ijzendoorn, 1999), including greater absorption - "the disposition for having episodes of 'total' attention that fully engage one's representational... resources" 


\section{ATTACHMENT \& PSYCHEDELIC MYSTICAL EXPERIENCE}

(Tellegen \& Atkinson, 1974, p. 268). Moreover, studies have found associations between disorganization and altered spiritual states, such as mystical experiences (Granqvist, Hagekull et al., 2012) and New Age spirituality (Granqvist et al., 2009), which are mediated by absorption.

An outstanding question concerns whether and how religious/spiritual experiences may constitute corrective attachment-related experiences. One intriguing possibility is that some such experiences (e.g., feeling accepted and loved by and fully connected to God after profound "selfsurrender") might aid insecure people in revising negative IWMs and "earning" felt security (Granqvist, 2020). However, research has not tested this hypothesis, mostly due to the hardships of inducing and managing the "set and setting" of spiritual experiences (Granqvist et al., 2005).

\section{Psychedelic Science}

In the following sections, we give a brief overview of psychedelic science, emphasizing the role of subjective phenomenological experiences for clinical outcomes. Finally, we delineate how, according to the REBUS model, psychedelics yield such experiences.

\section{Review of Central Findings}

Studies in the recent wave of psychedelic research have focused both on clinical trials of psychedelic-assisted psychotherapy and naturalistic psychedelic use. Though people use psychedelics in various settings, we primarily focus on psychedelic-assisted therapy here due to the crucial role of a controlled environment and experienced therapist (Carhart-Harris et al., 2018). Clinical studies indicate that the therapeutic use of psychedelics can effectively reduce symptoms associated with a long list of conditions, including anxiety, substance dependence, major depressive disorder, and suicidality (for a meta-analysis, see Luoma et al., 2020).

Individuals' experiences with psychedelics partly depend on contextual, extrapharmacological factors labeled "set and setting" (Zinberg, 1984). Set refers to the personality, 


\section{ATTACHMENT \& PSYCHEDELIC MYSTICAL EXPERIENCE}

intentions, expectations, and preparation of the person having the experience, and setting represents the physical, social, and cultural context in which the experience takes place (Hartogsohn, 2016). Whereas the use of psychedelics may lead to adverse effects (e.g., acute distress, enduring psychological issues; NIDA, 2019), there is a growing understanding of how to reduce harm (Johnson et al., 2008). Generally, adverse effects are associated with feelings of vulnerability during the experience and with the absence of perceived protection and support (Aday et al., 2021). Therefore, when consumed in a safe environment, psychedelics may result in transdiagnostic psychological benefits e.g., via improvements in attitudes toward self and others, distress tolerance, flexibility, openness, and mindfulness (e.g., Kočárová et al., 2021).

On this basis, the vast majority of protocols for psychedelic-assisted therapy include three phases: preparation, dosing, and integration (Johnson et al., 2008). In preparation sessions, therapists formulate a case conceptualization, provide psychoeducation about psychedelics and treatment, and establish a therapeutic alliance. During the dosing phase, participants ingest the compound with the continual support of at least one therapist, who, with minimal intervention, encourages clients to explore aspects of the psychedelic experience with openness. In the integration phase, therapists assist clients in reviewing their dosing experience, incorporating it into their personal narrative, and leveraging their insight to catalyze meaningful change.

\section{Role of Phenomenological Experiences in Psychedelic Treatment}

Qualitative studies have highlighted the positive implications of a person's subjective experience during and after the intake of psychedelics (Noorani et al., 2018). People often describe this experience as one of the most meaningful and transformative ones in their lives (Griffiths et al., 2018). The experience is often imbued with feelings of intense love and connectedness - directed at close others, humanity, and the world at large (Watts et al., 2017) - 


\section{ATTACHMENT \& PSYCHEDELIC MYSTICAL EXPERIENCE}

and a greater ability to engage with an expanded repertoire of emotions, including previously repressed distressing emotions, such as grief (Belser et al., 2017). People also report gaining new perspectives and deeper insight (e.g., into their habitual thoughts and coping methods), experiencing emotional breakthroughs (Roseman et al., 2019), or other sudden gains in clinically relevant phenomena (e.g., a shift in self-perception that reduces self-criticism and increases selfcompassion, Lafrance et al., 2017; see Breeksema et al., 2020, for a review).

Though most people describe positive experiences, challenging experiences (cf. "bad" trips) are not uncommon, including paranoid ideations and fear of losing sanity or dying (Gashi et al., 2021). Some studies have indicated that challenging experiences are related to less positive clinical outcomes of psychedelics (Roseman et al., 2019). However, if challenging experiences are processed and integrated into a coherent narrative, they may also be an important part of the therapeutic process (Barrett et al., 2016; Gashi et al., 2021).

Religious, spiritual, or mystical experiences (RSMEs) are also often experienced during the intake of psychedelics, even in controlled clinical trials (Griffiths et al., 2018). These experiences include a sense of transcendence, contact with the sacred, ultimacy, ego dissolution (loss of selfworld boundaries), and unity with an order that is far larger than oneself (e.g., God, higher power, the universe, ultimate reality; Yaden et al., 2017). RSMEs also tend to evoke positive feelings, such as love, safety, and security (Hood et al., 2018). RSMEs, including those induced by psychedelics, may reflect universalistic "core" spiritual experiences (cf. perennialism) or more particularistic experiences, co-constructed via culturally accessible (e.g., religious) interpretations (Brouwer \& Carhart-Harris, 2020). The way people process and integrate their psychedelic experiences depends on the interpretive lens provided by their metaphysical or religious beliefs (Granqvist, 2020). Whether or not interpreted religiously, RSMEs, especially 


\section{ATTACHMENT \& PSYCHEDELIC MYSTICAL EXPERIENCE}

those Stace (1960) called extrovertive states, are associated with relational themes such as a sense of connection and unity (Granqvist, 2020; Griffiths et al., 2018; Watts et al., 2017).

Studies have linked RSMEs to favorable clinical outcomes in psychedelic-assisted therapy (Andersen et al., 2021). Moreover, the degree of RSMEs, not the general intensity of the drug response, has uniquely predicted positive changes in attitudes, emotions, and social behavior (Griffiths et al., 2018). Importantly, as a predictor of clinical outcomes, RSMEs also outperform other subjective experiences, such as visual or auditory alterations (Roseman et al., 2018).

Psychedelic-induced RSMEs appear to affect changes in clinical symptoms via therapeutic gains in psychological traits that are otherwise relatively stable. For instance, RSMEs are linked to adaptive changes in the five-factor-model traits, such as post-therapy increases in openness to experience, agreeableness, and extraversion, and decreases in neuroticism (MacLean et al., 2011; Weiss et al., 2021). RSMEs also potentiate deeper insight and emotional breakthroughs, which seem to underlie positive outcomes of psychedelic-assisted therapy (Noorani et al., 2018).

Yet, as might be expected given the materialist Weltanschauung associated with medical science, some scholars argue that psychedelic-induced RSMEs are epiphenomenal byproducts of the neurobiological mechanisms that allegedly explain psychedelics' therapeutic effects (Olson, 2021). They note that other compounds acting on the serotonin $2 \mathrm{~A}$ system, such as $3,4-$ methylenedioxymethamphetamine (MDMA; albeit indirectly), show therapeutic potential without producing RSMEs (Mitchell et al., 2021). Ketamine too yields positive effects when given to unconscious patients (Kudoh et al., 2002). While other processes are also relevant, we tentatively follow the mounting evidence for a phenomenological position; RSMEs appear to facilitate the clinical utility of psychedelic-assisted therapy (Yaden \& Griffiths, 2021). 


\section{How Do Psychedelic Substances Work? The REBUS Model and Subjective Effects}

The REBUS ("RElaxed Beliefs Under pSychedelics") model provides the prevailing view of brain activity caused by psychedelics (Carhart-Harris \& Friston, 2019). The model accords with the predictive coding framework (Clark, 2016), positing that the brain's main function is to encode predictions (i.e., priors) of its environment, with minimal discrepancy, to capture and explain incoming stimuli (internal and external). Priors stem from experience (whether ontogenetic or phylogenetic), can be adjusted when novel or surprising information is encountered, are hierarchically nested, and operate at multiple levels of functional organization. The lowest level priors work on sensory input, while the highest work on a more abstract level, e.g., encoding high-level beliefs about self, others (including e.g., 'God') and the world. Highlevel priors are encoded by the functional organization and activity of brain systems that are structurally distal to sensory cortices such as the Default Mode Network (DMN; Margulies et al., 2016) and other high-level transmodal frontoparietal systems. Serotonin $2 \mathrm{~A}$ receptors (the main target of psychedelics) are spread across the cortex but are especially concentrated in these transmodal associative regions (Beliveau et al., 2017).

The REBUS model suggests that psychedelics (a) increase neural entropy (where increased entropy implies increased 'uncertainty' or 'randomness'), (b) transcend pre-established functional neural communication pathways, and (c) enable novel neural communications to occur (Carhart-Harris \& Friston, 2019). Thus, these substances have an entropic effect on spontaneous cortical activity (Schartner et al., 2017), and they transiently desynchronize activity in high-level regions such as nodes of the DMN, which likely disrupts the inhibitory constraints on up-stream information (e.g., from intrinsic sources like the limbic system; Ly et al., 2018) and relaxes the precision weighting of high-level priors - e.g., as indexed by decreased top-down 


\section{ATTACHMENT \& PSYCHEDELIC MYSTICAL EXPERIENCE}

travelling waves (Alamia et al., 2020). In this way, top-down, constraining, high-level beliefs are relaxed, enabling 'bottom-up' sensory and affective signals to register more freely in conscious experience (Lepow et al., 2021). Both dysregulated high-level cognition and facilitated affectivity converge in what has been labeled "pivotal mental states" -- states of facilitated "fast and deep' learning (and increased plasticity) that are the requisite for fast and deep (and potentially enduring) psychological transformations (Brouwer \& Carhart-Harris, 2021).

\section{Attachment, Psychedelics, and the Psychology of Spirituality/Religion}

In the following sections, we attempt to synthesize the REBUS model with attachment theory and the IWMs concept. Based on this synthesis, we then outline the three key proposals of attachment as predictor, outcome, and process in psychedelic-assisted therapy. Finally, we discuss the role of religion and spirituality for psychedelic experience and integration.

\section{Attachment and RSMEs in the REBUS model}

"The use to which a model in the brain is put is to transmit, store and manipulate information that helps in making predictions" (italics added; Bowlby, 1982, p. 80).

Though the theory of predictive coding is fairly recent, the key idea of the mind as predicting internal and external stimuli is one of the core tenets of attachment theory. Bowlby (1973) described how interactions in close, formative relationships crystallize into IWMs of the self and others, which influence patterns of socio-emotional functioning and beliefs about selfrelated experiences and others' behaviors. As such, IWMs may be viewed as experientially informed priors (particularly in early life, during sensitive developmental phases) for high-level information processing, which affect predictions and interpretations of interpersonally relevant stimuli. Like other priors, the influence and malleability of IWMs depend on contextual factors. The increased neuronal plasticity associated with psychedelics (i.e., relaxed priors) may allow 


\section{ATTACHMENT \& PSYCHEDELIC MYSTICAL EXPERIENCE}

for the reweighting of IWM-priors and potentially 'corrective' attachment experiences. Indeed, genes that affect the binding potential of serotonin 2A receptors moderate the influence of parental nurturance on child attachment behaviors (Salo et al., 2011), suggesting that the target receptor system of psychedelics may play a natural role in the formation and reweighting of attachment-related priors from the very outset of development.

To describe religion-as-attachment within the REBUS model, we posit that IWMs of attachment - with humans and God - are high-level priors with powerful effects on affective, social, and behavioral functioning (Granqvist, 2020). The intake of psychedelics and the initial subjective experience of entering the unknown, constitute a challenging, often distress-inducing situation (Roseman et al., 2019), which automatically activates the attachment system and associated IWMs. While this initial uncertainty may initially lead to an agitation of top-down processes and high-level IWMs, with psychological support, the unfolding of the psychedelic experience may bring such defensive strategies into conscious awareness, and ultimately reduce their influence and allow them to be revised. Naturally, because individual variations in attachment security (with humans and 'God') are intimately linked with the quality of IWMs/priors, the psychedelic experience can be expected to unfold differently depending on attachment security - how attachment manifests and is managed within the therapeutic relationships that form during a psychedelic therapy process.

\section{Attachment Security as a Predictor of Psychedelic-Related Experiences and Integration}

We hypothesize that attachment security is associated with both the quality of psychedelicinduced subjective experiences and the exploration and integration of these experiences. The quality of these experiences relies on individuals' ability to "surrender" to the drugs' effects and "let go" (Aday et al., 2021), and felt security can sustain such an ability. As reviewed above, 


\section{ATTACHMENT \& PSYCHEDELIC MYSTICAL EXPERIENCE}

attachment-secure people hold inner senses of self-worth and self-fortitude that allow them to remain calm, confident, and experientially open when facing uncertainty and ambiguity (Mikulincer \& Shaver, 2016). Moreover, they rely on effective emotion regulation strategies allowing them to mitigate distress and remain mindful, aware of, and fully engaged with, novel and exciting experiences even if they evoke temporary confusion (Mikulincer et al., 2003). As a result, attachment security may facilitate a calm surrender to the psychedelic-induced experience. By contrast, as reviewed in a previous section, attachment insecurities hinder effective distress management, which, in turn, precludes experiential openness, mindful awareness, and full engagement with novel and challenging experiences (Mikulincer \& Shaver, 2016). Attachment avoidance is related to cognitive and experiential closure, discomfort with novelty and ambiguity, and low tolerance of uncertainty (e.g., Mikulincer, 1997), which, in turn, may prevent "surrendering" to psychedelic-induced experiences. The intake of psychedelics may also lead avoidant individuals to rely on their typical defensive strategies, such as suppressing or escaping uncomfortable sensations, feelings, or thoughts, further exacerbating their resistance to "surrendering" to the drugs' effects. However, these avoidant defenses tend to fail in situations of extreme stress that involve a high cognitive or emotional load, leaving the avoidant person vulnerable to an overwhelming torrent of negative feelings and thoughts (Mikulincer et al., 2004) potentially alongside a 'psychedelic-like' altered state of consciousness (Brouwer \& CarhartHarris, 2021). This could also be the case for psychedelic-induced experiences: Psychedelics' dysregulatory action on top-down controlling processes might interfere with psychological defenses, such that suppressed material may resurge into awareness and create an aversive experiential state. This view of the action of psychedelics explains why such emphasis is placed on the importance of twinning the drugs with psychological support (Carhart-Harris et al., 2018). 


\section{ATTACHMENT \& PSYCHEDELIC MYSTICAL EXPERIENCE}

Without psychological support, attachment avoidant people may struggle to surrender to the drugs' effects, and ultimately the quality of their psychedelic-induced experience may be painful and disorganizing.

Attachment anxiety is linked to urgent needs for connection with a powerful source of protection and support, upregulation of distress, and a generalized, uncontrollable flow of negative sensations, feelings, and cognitions (Mikulincer \& Shaver, 2016). In the context of psychedelic use, attachment-anxious people's intense desire to be loved and cared for may be associated with a readiness to experience the drugs' effects - to let go or surrender (Aday, 2021), and experience union with an "external rescuer," thereby increasing the propensity for RSMEs (Granqvist, 2020). However, attachment-anxious people's problems with emotion regulation may negatively bias the quality of the psychedelic-induced experience, coloring it with intense mental pain and suffering. Notably, attachment anxiety may intensify the influence of the set and setting of psychedelic use; provided a supportive environment, attachment-anxious individuals may be able to engage openly with and perhaps benefit from the psychedelic experience (Carhart-Harris et al., 2018). It is also intriguing to speculate that attachment-anxious people may be more likely to appraise their psychedelic experience as having been 'saved' or 'redeemed,' which may lead to rapid, extreme, and potentially precarious transformations following psychedelic experiences underscoring the importance of grounding integration.

As noted above, attachment disorganization is associated with a proclivity for dissociative and absorbing mental states (Hesse \& van IJzendoorn, 1999), which has been found to contribute to altered states of consciousness and RSMEs, including those induced by psychedelics (Haijen et al., 2018; Granqvist, 2020). On this basis, attachment disorganization may facilitate more intense acute effects of psychedelics, including RSMEs, which might be mediated by heightened 


\section{ATTACHMENT \& PSYCHEDELIC MYSTICAL EXPERIENCE}

absorption. As noted by Stauffer et al. (2021, p. 529), "similar to attachment anxiety, the capacity for dissociation might actually prime an individual for the healing potential of non-ordinary states of consciousness" (cf. Hilgard's [1977|1986] notion of reaggregating, as opposed to disintegrating, dissociative states). However, the link between disorganized attachment and severe forms of dissociation may put a person at risk of overly intense, challenging psychedelicinduced RSMEs (e.g., Otto's [1925] "tremendum" and other fearful states) that may be difficult to integrate. Indeed, disorganization has been observed to interfere with therapeutic alliance and is associated with poorer therapeutic outcomes (Levy et al., 2018), in addition to a generally heightened risk for psychopathology (Bakermans-Kranenburg et al., 2009).

Attachment security may also facilitate the exploration/integration of psychedelic-induced experiences. Psychotherapy research has linked attachment security to better therapeutic processes and outcomes (Mikulincer et al., 2013). In a self-exacerbating cycle, secure attachment contributes to the formation of a stable therapeutic alliance and sustains open exploration, deep reflection, and coherent integration of personal memories, feelings, and beliefs, which, in turn, boosts attachment security over the course of treatment. Likewise, attachment security may aid in deriving enduring, transformational meaning from powerful experiences induced by psychedelics. While insecure attachment may hinder this process, as a maladaptive "set" factor, a sound "setting" - a supportive therapeutic environment and competent therapist - may provide the compensatory security necessary to facilitate more favorable psychedelic experiences and their integration into a person's narrative and IWMs of the self and others.

In the only psychedelic study to date directly informed by attachment theory, Stauffer et al. (2021) conducted a pilot pre-post study of 18 long-term AIDS survivors receiving a one-dose psilocybin-assisted group therapy for demoralization. In line with our proposals, dispositional 


\section{ATTACHMENT \& PSYCHEDELIC MYSTICAL EXPERIENCE}

attachment orientations measured at baseline strongly predicted patients' subjective experiences: Whereas attachment anxiety predicted more mystical experiences, attachment avoidance predicted more challenging (distress-eliciting) experiences.

\section{Attachment Security as a Clinical Outcome}

Addressing clients' attachment security may also be a worthwhile clinical aim of psychedelic-assisted therapy. Attachment security has been recognized as a key "nonspecific factor" in psychotherapy across treatment modalities, and increasing attachment security has also emerged as a meaningful therapeutic outcome in and of itself (Levy et al., 2018). Although IWMs are relatively core high-level priors, the intake of psychedelics and the ensuing increase in brain connectivity/entropy may relax their integrity and top-down constraining influence and create the possibility for novel experiences that, with nurturing psychological support, could lead to favorable revisions of these priors. Such "improvements" in attachment representations may underlie the acute RSMEs and pivotal life transformations people often attribute to psychedelic experiences (Brouwer \& Carhart-Harris, 2021).

Reports of psychedelic-induced subjective experiences appear to support this idea. These experiences, while often acutely challenging, have been found to trigger subsequent emotional and cognitive changes indicative of improved felt security, including improved self-perception, increased felt interconnectedness and experiential and emotional openness (Breeksema et al., 2020). Moreover, Holze et al. (2021) found that, at close to peak plasma concentrations of LSD (200 mcg), participants displayed higher empathy and greater release of oxytocin (a hormone that presumably facilitates attachment/bonding) - an effect that subsided (for oxytocin) as subjective drug effects subsided at 8 hours post administration. By putatively enhancing felt security, psychedelic-assisted therapy can set in motion relearning processes that not only 


\section{ATTACHMENT \& PSYCHEDELIC MYSTICAL EXPERIENCE}

alleviate symptoms but continue to impact clinical outcomes beyond the end of treatment (Mikulincer et al., 2013). Thus, psychedelic-assisted therapy may be able to facilitate durable, clinically favorable changes via catalyzing relearning processes linked to attachment and IWMs.

Stauffer et al. (2021) found preliminary evidence that psychedelic-assisted therapy promotes attachment security. Clients' self-reports of attachment anxiety significantly decreased following psilocybin-assisted group therapy ( $d=.45$ from baseline to 3 -months follow-up). Attachment avoidance seemed to decrease as well $(d s=.28$ and .19 , at 3 weeks and 3 months post-treatment, respectively), but these changes did not reach statistical significance. While statistical power was low $(n=18)$ and the study lacked a control group, the results suggest that psychedelic-assisted therapy may enhance attachment security. It is intriguing to speculate that, though attachment avoidance may be the more challenging clinical presentation, attachment avoidance could also be improved via psychedelic-assisted therapy, perhaps requiring an alternative therapeutic model (Zeifman \& Wagner, 2020).

\section{Attachment-Related Dynamics as Process-Level Mechanisms}

A third way in which attachment theory can contribute to psychedelic science is by informing about the psychological and interpersonal processes involved in psychedelic experiences and process-level (or proximal) mechanisms of clinical outcomes. At its core, attachment theory describes perceptual, affective, cognitive, and behavioral reactions to distressing experiences aimed at restoring felt security as well as individual differences in these reactions based on high-level IWMs or priors (Mikulincer \& Shaver, 2016).

By stimulating serotonin $2 \mathrm{~A}$ receptors, psychedelics engage the same circuitry that is presumably involved in the formation of IWMs earlier in development (e.g., associative learning of threat-related and safety cues; Murnane, 2019), much like how stress can increase 


\section{ATTACHMENT \& PSYCHEDELIC MYSTICAL EXPERIENCE}

neuroplasticity (Brivio et al., 2020) and accelerate learning rate (Harvey, 2003) in a manner consistent with the reopening of critical periods (Lepow et al., 2021), which may enable the relearning of attachment related IWMs. Specifically, psychedelics may activate the attachment system due to the loss of control and triggering of powerful threat-related emotions. Moreover, relaxing the precision weighting of relevant IWM priors, psychedelics may reduce the influence of insecure, defensive IWMs, opening them up to healthy revision. In this way, psychedelics create opportunities to revise presently maladaptive (insecure) IWMs and extinguish learned expectations of lack of comfort or abandonment or hostility in stressful situations (Bosmans et al., 2019). These sudden gains in felt security may occur during an acute experience that promotes openness and flexibility toward external and internal stimuli. The result may be a radical improvement in expectations of comfort and security, or what Main et al. (1985) called a "corrective attachment experience," by producing a profound experience of being connected with and embraced by a powerful benevolent figure (e.g., God, nature, the universe). That is, psychedelic-induced experiences of unity and transcendence may facilitate spiritual corrective attachment experiences, likely contingent on the quality of the accompanying support).

A frequently reported effect of psychedelics is greater access to distressing emotions, thoughts, and memories (Healy, 2021). While internal stimuli may retain their painful quality, with adjacent psychological support, psychedelics can be used to address and overcome experiential avoidance, to enable a closer contact with inner experiences and deeper processing of all types of feeling states. Over time, these improvements in mindfulness-related phenomena may improve distress tolerance and behavioral activation for valued ends (Watts et al., 2017).

Finally, psychedelic-assisted therapy is conducted with a trained, experienced therapist capable of providing a "holding environment" (Tai et al., 2021) with the therapist, "guide" or 


\section{ATTACHMENT \& PSYCHEDELIC MYSTICAL EXPERIENCE}

“trip sitter” who cares for a vulnerable person's well-being in a largely observant, noninterventional way (Badiner \& Grey, 2015). Psychedelics' activation of the attachment system catalyzing a potential healthy recalibration of the weighting of priors (e.g., insecure IWMs) may facilitate corrective emotional experiences with the therapist. Psychotherapy research consistently shows the quality of client-therapist relationship (i.e., therapeutic alliance) to be one the strongest predictors of clinical outcomes regardless of treatment modality (Horvath et al., 2011), and this has recently also been shown with psychedelics (Murphy et al., in press). Therefore, we can expect that the quality of client-therapist relationship, in general, and the extent to which a therapist is perceived as a reliable safe haven and secure base, in particular, are key aspects of a positive set/setting, conducive to positive therapeutic outcome. However, while clients in psychedelic-assisted therapy may experience their therapist as a source of comfort and support, they typically attribute the curing effects to intrapsychic processes and unity-type mystical experiences (Watts et al., 2017). One should also be mindful of the potential risk of problematic patient-therapist attachment formations both during a therapeutic process and, perhaps most importantly, afterwards where the ending of particularly intense therapeutic relationships may be psychologically triggering, if not managed carefully. Relatedly, the risk (as well as the utility or value) of enhanced transference formations via psychedelics should also be considered.

\section{Role of Religion/Spirituality in Psychedelic Experience and Integration}

The effect of psychedelic-induced RSMEs may depend on the influence of individuals' preexisting religious/spiritual beliefs and practices. For example, especially in monotheistic faiths, feelings of transcendence and unity may be interpreted as (or attributed to) direct experiences of encountering God. In other settings, the same underlying (neural) state could be attributed to 


\section{ATTACHMENT \& PSYCHEDELIC MYSTICAL EXPERIENCE}

unity with nature (cf. "nature mysticism"), and in yet others to "going insane," "losing one's mind," or "becoming delusional." However, we are aware of no research systematically addressing whether such different worldview-informed interpretations are differentially linked to the subjective effects of or clinical outcomes from psychedelic use.

The religion-mental health link is relevant to RSMEs and their continued impact. Research consistently highlights that religion is associated with better mental health presentations, albeit in complex ways. Attachment security to God, a perceived personal bond with God, and a sense of unity with God have been highlighted as pivotal mediators of this association (Koenig et al., 2012), and the community component of religious practice may also play an important role.

Since religions address areas of ultimate concern, religiosity can be intertwined with symptoms, coping resources, and life events, and a majority of psychotherapy clients want to raise religious issues in therapy (Koenig, 2018). On this basis, several evidence-based spiritually integrated treatment models have been developed (Rosmarin, 2018). Unfortunately, there is little material available on spirituality-guided integration of psychedelic-induced RSMEs (cf. Badiner \& Grey, 2015). A potential focus for research is spiritual struggles - tensions, strains, and conflicts concerning what people hold sacred (Exline, 2013). Whereas these struggles are associated with emotional distress, exploring and processing them seems to contribute to heightened well-being (Exline, 2013). Therefore, the arousal of spiritual struggles during psychedelic-induced RSMEs can be explicitly explored and elaborated during the integration phase, and this deep processing may produce impactful positive transformations.

\section{Future Directions and Concluding Remarks}

We have outlined how the synthesis of attachment theory with the REBUS model can serve as an integrating heuristic framework for a psychedelic science of spirituality and religion. 


\section{ATTACHMENT \& PSYCHEDELIC MYSTICAL EXPERIENCE}

This integrative framework aids psychedelic science in identifying key predictors, outcomes, and process-level mechanisms pertinent to psychedelic-induced RSMEs and their integration. It also explains how psychedelics provide attachment-insecure people with spiritual/religious corrective attachment experiences that may enhance their felt security with regard to God and humans.

Psychedelic science can benefit greatly from this cross-fertilization. An attachment theory perspective may spur methodological advancements in psychedelic science by offering a robust research paradigm, a coherent structure for generating hypotheses, and rigorously developed measures. In addition, the field of psychedelic science is still establishing the set-and-setting factors associated with favorable psychedelic experiences (Aday, 2021). Though the empirical evidence is still preliminary, attachment theory is a promising framework for identifying salient candidate factors, which may, in turn, bolster the effectiveness of psychedelic-assisted therapy.

Similarly, the psychology of religion and spirituality can benefit from an attachmentinformed psychedelic science. In particular, psychedelic research may present a new avenue of research on religious and spiritual change. We described the compensation pathway, whereby individuals turn to God and religion in the absence of satisfactory human attachment figures, which tends to lead to sudden, intense religious changes that are ultimately characterized by emotional turbulence, spiritual struggles, and interpersonal conflicts. In contrast, by construing a safe, comfortable, and supportive setting, psychedelics may yield religious and spiritual changes that, albeit sudden, result in a more secure path forward. Relatedly, psychedelic research presents an intriguing opportunity to explore the possibility that individuals with insecure attachment can, via psychedelic-assisted therapy, ultimately attain "earned secure attachment" (Main et al., 2003). Such research can also investigate whether earned security unfolds via corrective relational experiences with God, therapists, or others. 


\section{ATTACHMENT \& PSYCHEDELIC MYSTICAL EXPERIENCE}

Our attachment-REBUS synthesis may, in addition, contribute to protocols for guiding psychedelic use and facilitating integration by highlighting process-level mechanisms for favorable clinical outcomes. Attachment considerations may also guide users who seek to express themselves spiritually during a psychedelic session (e.g., religious meditation) and integrate their experience with religious meaning. Psychedelics and associated spiritual attachment-relevant experiences may be particularly relevant to parts of life laden with a quest for religious significance (e.g., end-of-life care; see Margolin \& Hartman, 2021).

Furthermore, psychedelic science could draw on recent developments in psychotherapy research focusing on "sudden gains" in clinical outcomes. Psychedelic experiences may catalyze dramatic changes in attachment security, among other consequential transdiagnostic outcomes. In addition, studying clients' attachment to the therapist would shed light on the interpersonal dynamics of psychedelic-assisted therapy and highlight the therapist's role as part of an effective setting for bringing about positive clinical outcomes.

Based on Bowlby's (1982) depiction of experientially based IWMs as aiding in making predictions about the world, we have suggested that IWMs may be conceptualized as cognitive priors within a predictive coding framework. Admittedly, the attachment literature has not fully clarified the nature, organization, and stability of IWMs (Duschinsky, 2020) or their neurobiological correlates. Whereas IWMs escape direct observation, considering them in a predictive coding framework opens up new vistas to address some of the lingering questions about their stability and organization. One possibility to assess whether IWMs are relaxed during psychedelic sessions, which would support our framing of IWMs as priors, is to observe attachment behaviors before and during a psychedelic experience (e.g., emotional avoidance, engaging the therapist; Talia et al., 2017). Another would be to employ a measure of belief 


\section{ATTACHMENT \& PSYCHEDELIC MYSTICAL EXPERIENCE}

confidence, given that felt 'confidence' is considered the psychological counterpart of 'precision weighting.' This approach is appealing, as confidence ratings (pre, post, and during intervention) could be applied to any belief - including e.g., beliefs about self, others and 'God'.

Lastly, we have spotlighted RSMEs as they are among the strongest, most replicated predictors of favorable clinical outcomes in psychedelic-assisted therapy (Yaden \& Griffiths, 2021). However, we acknowledge that other experiential states, such as emotional breakthrough and psychological insight, might also be influential therapeutic factors even in the absence of RSMEs (Letheby, 2021). Moreover, some have challenged whether subjective experiences induced by psychedelics are needed for their clinical utility (Olson, 2021).

In sum, we have synthesized attachment theory with the predictive coding-inspired REBUS model. Based on this synthesis, we propose that attachment security predicts psychedelic experiences and their subsequent outcomes. With effective psychological integration, psychedelic-assisted therapy may be a particularly powerful method for facilitating increased attachment security. Indeed, attachment theory may help identify and explain processlevel mechanisms relevant to psychological change seen with psychedelics. Its power to explain specific phenomena such as RSMEs and personal relations to 'God' may be considerable. We suggest that reference to attachment theory may help enhance and advance psychedelic science and potentially maximize clinical outcomes associated with psychedelic-assisted therapy. 


\section{References}

Aday, J. S., Davis, A. K., Mitzkovitz, C. M., Bloesch, E. K., \& Davoli, C. C. (2021). Predicting reactions to psychedelic drugs: A systematic review of states and traits related to acute drug effects. ACS Pharmacology \& Translational Science, 4(2), 424-435.

Alamia, A., Timmermann, C., Nutt, D. J., VanRullen, R., \& Carhart-Harris, R. L. (2020). Correction: DMT alters cortical travelling waves. eLife, 9, e64623.

Andersen, K. A., Carhart-Harris, R., Nutt, D. J., \& Erritzoe, D. (2021). Therapeutic effects of classic serotonergic psychedelics: A systematic review of modern-era clinical studies. Acta Psychiatrica Scandinavica, 143(2), 101-118.

Badiner, A. \& Grey, A. (Eds.). (2015). Zig zag zen: Buddhism and psychedelics. Synergetic Press.

Bakermans-Kranenburg, M., \& van IJzendoorn, M. H. (2009). The first 10,000 Adult Attachment Interviews: Distributions of adult attachment representations in clinical and non-clinical groups. Attachment and Human Development, 11(3), 223-263.

Barrett, F. S., Bradstreet, M. P., Leoutsakos, J. M. S., Johnson, M. W., \& Griffiths, R. R. (2016). The Challenging Experience Questionnaire: Characterization of challenging experiences with psilocybin mushrooms. Journal of Psychopharmacology, 30(12), 1279-1295.

Beliveau, V., Ganz, M., Feng, L., Ozenne, B., Højgaard, L., Fisher, P. M., ... Knudsen, G. M. (2017). A high-resolution in vivo atlas of the human brain's serotonin system. Journal of Neuroscience, 37(1), 120-128.

Belser, A. B., Agin-Liebes, G., Swift, T. C., Terrana, S., Devenot, N., Friedman, H. L., ... Ross, S. (2017). Patient experiences of psilocybin-assisted psychotherapy: An interpretative phenomenological analysis. Journal of Humanistic Psychology, 57(4), 354-388. 
Bosmans, G., Waters, T. E. A., Finet, C., de Winter, S., \& Hermans, D. (2019). Trust development as an expectancy-learning process: Testing contingency effects. PLoS ONE, 14(12), e0225934.

Bowlby, J. (1973). Attachment and loss: Vol. 2 Separation anxiety and anger. Basic Books. Bowlby, J. (1982). Attachment and loss: Vol. 1 Attachment (2nd ed.). Basic Books.

Bowlby, J. (1988). A secure base: Clinical applications of attachment theory. Routledge.

Breeksema, J. J., Niemeijer, A. R., Krediet, E., Vermetten, E., \& Schoevers, R. A. (2020). Psychedelic treatments for psychiatric disorders: A systematic review and thematic synthesis of patient experiences in qualitative studies. CNS Drugs, 34(9), 925-946.

Brennan, K. A., Clark, C. A., \& Shaver, P. R. (1998). Self-report measurement of adult attachment: An integrative overview. In J. A. Simpson \& W. S. Rholes (Eds.), Attachment theory and close relationships (pp. 46-76). Guilford.

Brivio, P., Sbrini, G., Riva, M. A., \& Calabrese, F. (2020). Acute stress induces cognitive improvement in the novel object recognition task by transiently modulating Bdnf in the prefrontal cortex of male rats. Cellular and Molecular Neurobiology, 40, 1037-1047.

Brouwer, A., \& Carhart-Harris, R. L. (2021). Pisvotal mental states. Journal of Psychopharmacology, 35(4), 319-352.

Bruhn, J. G., De Smet, P. A. G. M., El-Seedi, H. R., \& Beck, O. (2002). Stigma of mental illness in Mescaline use for 5700 years. The Lancet, 359(9320), 1866.

Carhart-Harris, R. L., \& Friston, K. J. (2019). REBUS and the anarchic brain: Toward a unified model of the brain action of psychedelics. Pharmacological Reviews, 71(3), 316-344. 
ATTACHMENT \& PSYCHEDELIC MYSTICAL EXPERIENCE

Carhart-Harris, R. L., Roseman, L., Haijen, E., Erritzoe, D., Watts, R., Branchi, I., \& Kaelen, M. (2018). Psychedelics and the essential importance of context. Journal of Psychopharmacology, 32(7), 725-731.

Cherniak, A. D., Mikulincer, M., Shaver, P. R., \& Granqvist, P. (2021). Attachment theory and religion. Current Opinion in Psychology, 40, 126-130.

Clark, A. (2016). Surfing uncertainty. Oxford University Press.

Davis, E. B., Kimball, C. N., Aten, J. D., Andrews, B., Van Tongeren, D. R., Hook, J. N., ... Park, C. L. (2019). Religious meaning making and attachment in a disaster context: A longitudinal qualitative study of flood survivors. Journal of Positive Psychology, 14(5), 659-671.

Duschinsky, R. (2020). Cornerstones of Attachment Research. Oxford University Press.

Exline, J. J. (2013). Religious and spiritual struggles. In K. I. Pargament (Ed.-in-Chief ), J. J. Exline, \& J. W. Jones (Assoc. Eds.), APA handbook of psychology, religion, and spirituality (Vol. 1, pp. 459-475). American Psychological Association.

Ferguson, M. A., Nielsen, J. A., King, J. B., Dai, L., Giangrasso, D. M., Holman, R., ... \& Anderson, J. S. (2018). Reward, salience, and attentional networks are activated by religious experience in devout Mormons. Social Neuroscience, 13(1), 104-116.

Fraley, R. C., \& Shaver, P. R. (2000). Adult romantic attachment: Theoretical developments, emerging controversies, and unanswered questions. Review of General Psychology, 4(2), 132-154.

Fraley, R. C., Vicary, A. M., Brumbaugh, C. C., \& Roisman, G. I. (2011). Patterns of stability in adult attachment: An empirical test of two models of continuity and change. Journal of Personality and Social Psychology, 101(5), 974-992. 


\section{ATTACHMENT \& PSYCHEDELIC MYSTICAL EXPERIENCE}

Gashi, L., Sandberg, S., \& Pedersen, W. (2021). Making "bad trips" good: How users of psychedelics narratively transform challenging trips into valuable experiences. International Journal of Drug Policy, 87, 102997.

Granqvist, P. (2020). Attachment in religion and spirituality: A wider view. Guilford Press.

Granqvist, P., Fransson, M., \& Hagekull, B. (2009). Disorganized attachment, absorption, and new age spirituality: A mediational model. Attachment and Human Development, 11(4), $385-403$

Granqvist, P., Fredrikson, M., Unge, P., Hagenfeldt, A., Valind, S., Larhammar, D., \& Larsson, M. (2005). Sensed presence and mystical experiences are predicted by suggestibility, not by the application of transcranial weak complex magnetic fields. Neuroscience Letters, $379(1), 1-6$.

Granqvist, P., Hagekull, B., \& Ivarsson, T. (2012). Disorganized attachment promotes mystical experiences via a propensity for alterations in consciousness (absorption). International Journal for the Psychology of Religion, 22(3), 180-197.

Granqvist, P., \& Kirkpatrick, L. A. (2004). Religious conversion and perceived childhood attachment: A meta-analysis. International Journal of Psychology of Religion, 14(4), 223 250.

Granqvist, P., \& Kirkpatrick, L. A. (2016). Attachment and religious representations and behavior. In J. Cassidy \& P. R. Shaver (Eds.), Handbook of attachment: Theory, research, and clinical applications (3rd ed., pp. 856-878). Guilford.

Granqvist, P., Mikulincer, M., Gewirtz, V., \& Shaver, P. R. (2012). Experimental findings on God as an attachment figure: Normative processes and moderating effects of internal working models. Journal of Personality and Social Psychology, 103(5), 804-818. 


\section{ATTACHMENT \& PSYCHEDELIC MYSTICAL EXPERIENCE}

Greenwald, Y., Mikulincer, M., Granqvist, P., \& Shaver, P. R. (2021). Apostasy and conversion: Attachment orientations and individual differences in the process of religious change. Psychology of Religion and Spirituality, 13(4), 425-436.

Griffiths, R. R., Richards, W. A., McCann, U., \& Jesse, R. (2006). Psilocybin can occasion mystical-type experiences having substantial and sustained personal meaning and spiritual significance. Psychopharmacology, 187(3), 268-283.

Griffiths, R. R., Johnson, M. W., Richards, W. A., Richards, B. D., Jesse, R., MacLean, K. A., ... Klinedinst, M. A. (2018). Psilocybin-occasioned mystical-type experience in combination with meditation and other spiritual practices produces enduring positive changes in psychological functioning and in trait measures of prosocial attitudes and behaviors. Journal of Psychopharmacology, 32(1), 49-69.

Haijen, E. C. H. M., Kaelen, M., Roseman, L., Timmermann, C., Kettner, H., Russ, S., ... Carhart-Harris, R. L. (2018). Predicting responses to psychedelics: A prospective study. Frontiers in Pharmacology, 9, 897.

Hartogsohn, I. (2016). Set and setting, psychedelics and the placebo response: An extrapharmacological perspective on psychopharmacology. Journal of Psychopharmacology. $30(12), 1259-1267$.

Harvey, J. A. (2003). Role of the serotonin 5-HT2A receptor in learning. Learning \& Memory, $10(5), 355-362$.

Healy, C. J. (2021). The acute effects of classic psychedelics on memory in humans. Journal of Psychopharmacology, 238(3), 639-653. 


\section{ATTACHMENT \& PSYCHEDELIC MYSTICAL EXPERIENCE}

Hesse, E., \& Van IJzendoorn, M. H. (1999). Propensities towards absorption are related to lapses in the monitoring of reasoning or discourse during the Adult Attachment Interview: A preliminary investigation. Attachment and Human Development, 1(1), 67-91.

Hilgard, E. R. (1986). Divided consciousness: Multiple controls in human thought and Action (2 ${ }^{\text {nd }}$ ed.). Wiley.

Holze, F., Avedisian, I., Varghese, N., Eckert, A., \& Liechti, M. E. (2021). Role of the 5-HT2A receptor in acute effects of LSD on empathy and circulating oxytocin. Frontiers in Pharmacology, 12, 711255 .

Hood, R. W. Jr., Hill, P. C., \& Spilka, B. (2018). The psychology of religion: An empirical approach (5th ed.). Guilford Press.

Horvath, A. O., Del Re, A. C., Flückiger, C., \& Symonds, D. (2011). Alliance in individual psychotherapy. In J. C. Norcross (Ed.), Psychotherapy relationships that work: Evidencebased responsiveness (pp. 25-69). Oxford University Press.

Johnson, M. W., Richards, W. A., \& Griffiths, R. R. (2008). Human hallucinogen research: Guidelines for safety. Journal of Psychopharmacology, 22(6), 603-620.

Johnson, M. W., Hendricks, P. S., Barrett, F. S., \& Griffiths, R. R. (2019). Classic psychedelics: An integrative review of epidemiology, therapeutics, mystical experience, and brain network function. Pharmacology and Therapeutics, 197, 83-102.

Kimball, C., Cook, K., Boyatzis, C., \& Leonard, K. (2013). Meaning making in emerging adults' faith narratives. Journal of Psychology and Christianity, 32(3), 221-233.

Kočárová, R., Horáček, J., \& Carhart-Harris, R. (2021). Does psychedelic therapy have a transdiagnostic action and prophylactic potential? Frontiers in Psychiatry, 12, 661233. 
ATTACHMENT \& PSYCHEDELIC MYSTICAL EXPERIENCE

Koenig, H. G. (2018). Religion and mental health: Research and clinical applications. Academic Press.

Koenig, H. G., King, D. E., \& Carson, V. B. (2012). Handbook of religion and health (2nd ed.). Oxford University Press.

Kudoh, A., Takahira, Y., Katagai, H., \& Takazawa, T. (2002). Small-dose ketamine improves the postoperative state of depressed patients. Anesthesia and Analgesia, 95(1), 114-118.

Lafrance, A., Loizaga-Velder, A., Fletcher, J., Renelli, M., Files, N., \& Tupper, K. W. (2017). Nourishing the spirit: Exploratory research on ayahuasca experiences along the continuum of recovery from eating disorders. Journal of Psychoactive Drugs, 49(5), 427-435.

Lepow, L., Morishita, H., \& Yehuda, R. (2021). Critical period plasticity as a framework for psychedelic-assisted psychotherapy. Frontiers in Neuroscience, 15, 710004.

Letheby, C. (2021). Philosophy of psychedelics. Oxford University Press.

Levy, K. N., Kivity, Y., Johnson, B. N., \& Gooch, C. V. (2018). Adult attachment as a predictor and moderator of psychotherapy outcome: A meta-analysis. Journal of Clinical Psychology, 74(11), 1996-2013.

Luoma, J. B., Chwyl, C., Bathje, G. J., Davis, A. K., \& Lancelotta, R. (2020). A meta-analysis of placebo-controlled trials of psychedelic-assisted therapy. Journal of Psychoactive Drugs, 52(4), 289-299.

Ly, C., Greb, A. C., Cameron, L. P., Wong, J. M., Barragan, E. V, Wilson, P. C., ... Olson, D. E. (2018). Psychedelics promote structural and functional neural plasticity. Cell Reports, 23(11), 3170-3182. 
MacLean, K. A., Johnson, M. W., \& Griffiths, R. R. (2011). Mystical experiences occasioned by the hallucinogen psilocybin lead to increases in the personality domain of openness. Journal of Psychopharmacology, 25(11), 1453-1461.

Main, M., Goldwyn, R., \& Hesse, E. (2003). Adult attachment scoring and classification system. Unpublished manuscript, University of California at Berkeley.

Main, M., Kaplan, N., \& Cassidy, J. (1985). Security in infancy, childhood, and adulthood: A move to the level of representation. Monographs of the Society for Research in Child Development, 50(1), 66-104.

Main, M., \& Solomon, J. (1990). Procedures for identifying infants as disorganized/disoriented during the Ainsworth strange situation. In M. T. Greenberg, D. Cicchetti, \& E. M. Cummings (Eds.), Attachment in the preschool years: Theory, research, and intervention (pp. 121-160). University of Chicago Press.

Margolin, M., \& Hartman, S. (2021, April 23). Jews, Christians, and Muslims are reclaiming ancient psychedelic practices, and that could help with legalization. Rolling Stone. https://www.rollingstone.com/culture/culture-features/psychedelics-religion-entheogens$1160408 /$

Margulies, D. S., Ghosh, S. S., Goulas, A., Falkiewicz, M., Huntenburg, J. M., Langs, G., ... \& Smallwood, J. (2016). Situating the default-mode network along a principal gradient of macroscale cortical organization. Proceedings of the National Academy of Sciences, 113(44), 12574-12579.

Mikulincer, M. (1997). Adult attachment style and information processing: Individual differences in curiosity and cognitive closure. Journal of Personality and Social Psychology, 72(5), 1217-1230. 
Mikulincer, M., Dolev, T., \& Shaver, P. R. (2004). Attachment-related strategies during thought suppression: Ironic rebounds and vulnerable self-representations. Journal of Personality and Social Psychology, 87(6), 940-956.

Mikulincer, M., Shaver, P. R., \& Pereg, D. (2003). Attachment theory and affect regulation: The dynamics, development, and cognitive consequences of attachment-related strategies. Motivation and Emotion, 27(2), 77-102.

Mikulincer, M., \& Shaver, P. R. (2016). Attachment in adulthood: Structure, dynamics, and change (2nd ed.). Guilford Press.

Mikulincer, M., Shaver, P. R., \& Berant, E. (2013). An attachment perspective on therapeutic processes and outcomes. Journal of Personality, 81(6), 606-616.

Mitchell, J. M., Bogenschutz, M., Lilienstein, A., Harrison, C., Kleiman, S., Parker-Guilbert, K., ... Doblin, R. (2021). MDMA-assisted therapy for severe PTSD: A randomized, doubleblind, placebo-controlled phase 3 study. Nature Medicine, 27(6), 1025-1033.

Murnane, K. S. (2019). Serotonin 2A receptors are a stress response system: implications for post-traumatic stress disorder. Behavioural Pharmacology, 30(2 and 3-Spec Issue), 151162.

Murphy, R., Kettner, H., Zeifman, R., Girabaldi, B., Kartner, L., Martell, J., Read, T., MurphyBeiner, A., Baker-Jones, M., Nutt, D., Erritzoe, D., Watts, R., \& Cahart-Harris, R. (in press). Therapeutic alliance and rapport modulates responses to psilocybin assisted therapy. Frontiers of Pharmacology.

NIDA. (2019, April 22). Hallucinogens DrugFacts. https://www.drugabuse.gov/publications/drugfacts/hallucinogens 
Noorani, T., Garcia-Romeu, A., Swift, T. C., Griffiths, R. R., \& Johnson, M. W. (2018). Psychedelic therapy for smoking cessation: Qualitative analysis of participant accounts. Journal of Psychopharmacology, 32(7), 756-769.

Olson, D. E. (2021). The subjective effects of psychedelics may not be necessary for their enduring therapeutic effects. ACS Pharmacology and Translational Science, 4(2), 563-567.

Otto, R. (1925). The idea of the holy. Oxford University Press.

Paloutzian, R. F., \& Park, C. L. (Eds.) (2013). Handbook of the psychology of religion and spirituality (2nd ed.). Guilford Press.

Pirutinsky, S., Cherniak, A. D., \& Rosmarin, D. H. (2020). Implicit and explicit attitudes towards God and life satisfaction. Psychology of Religion and Spirituality, 12(4), 387-392.

Roseman, L., Haijen, E., Idialu-Ikato, K., Kaelen, M., Watts, R., \& Carhart-Harris, R. (2019). Emotional breakthrough and psychedelics: Validation of the Emotional Breakthrough Inventory. Journal of Psychopharmacology, 33(9), 1076-1087.

Roseman, L., Nutt, D. J., \& Carhart-Harris, R. L. (2018). Quality of acute psychedelic experience predicts therapeutic efficacy of psilocybin for treatment-resistant depression. Frontiers in Pharmacology, 8, 974.

Rosmarin, D. H. (2018). Spirituality, religion, and cognitive-behavioral therapy: A guide for clinicians. Guilford Press.

Salo, J., Jokela, M., Lehtimäki, T., \& Keltikangas-Järvinen, L. (2011). Serotonin receptor 2A gene moderates the effect of childhood maternal nurturance on adulthood social attachment. Genes, Brain and Behavior, 10(7), 702-709. 
Schartner, M. M., Carhart-Harris, R. L., Barrett, A. B., Seth, A. K., \& Muthukumaraswamy, S. D. (2017). Increased spontaneous MEG signal diversity for psychoactive doses of ketamine, LSD and psilocybin. Scientific reports, 7, 46421.

Schjoedt, U., Stødkilde-Jørgensen, H., Geertz, A. W., \& Roepstorff, A. (2009). Highly religious participants recruit areas of social cognition in personal prayer. Social Cognitive and Affective Neuroscience, 4(2), 199-207.

Sharp, C. A., Davis, E. B., George, K., Cuthbert, A. D., Zahl, B. P., Davis, D. E., ... Aten, J. D. (2019). Measures of God representations: Theoretical framework and critical review. Psychology of Religion and Spirituality, 13(3), 340-357.

Stace, W. T. (1960). Mysticism and philosophy. St. Martin's Press.

Stauffer, C. S., Anderson, B. T., Ortigo, K. M., \& Woolley, J. (2021). Psilocybin-assisted group therapy and attachment: Observed reduction in attachment anxiety and influences of attachment insecurity on the psilocybin experience. ACS Pharmacology and Translational Science, 4(2), 526-532.

Stovall-McClough, K. C., \& Dozier, M. (2016). Attachment states of mind and psychopathology in adulthood. In J. Cassidy \& P. R. Shaver (Eds.), Handbook of attachment: Theory, research, and clinical applications (3rd ed., pp. 715-738). Guilford Press.

Tai, S. J., Nielson, E. M., Lennard-Jones, M., Johanna Ajantaival, R. L., Winzer, R., Richards, W. A., ... Malievskaia, E. (2021). Development and evaluation of a therapist training program for psilocybin therapy for treatment-resistant depression in clinical research. Frontiers in Psychiatry, 12, 586682. 
Talia, A., Miller-Bottome, M., \& Daniel, S. I. F. (2017). Assessing attachment in psychotherapy: Validation of the Patient Attachment Coding System (PACS). Clinical Psychology and Psychotherapy, 24(1), 149-161.

Tellegen, A., \& Atkinson, G. (1974). Openness to absorbing and self-altering experiences ("absorption"), a trait related to hypnotic susceptibility. Journal of Abnormal Psychology, 83(3), 268-277.

Watts, R., Day, C., Krzanowski, J., Nutt, D., \& Carhart-Harris, R. (2017). Patients' accounts of increased "connectedness" and "acceptance" after psilocybin for treatment-resistant depression. Journal of Humanistic Psychology, 57(5), 520-564.

Weiss, B., Miller, J. D., Carter, N.T., \& Campbell, K.W. (2021). Examining changes in personality following shamanic ceremonial use of ayahuasca. Scientific Reports, 11, 1-15.

Yaden, D. B., \& Griffiths, R. R. (2021). The subjective effects of psychedelics are necessary for their enduring therapeutic effects. ACS Pharmacology and Translational Science, 4(2), 568-572.

Yaden, D. B., Haidt, J., Hood, R. W., Vago, D. R., \& Newberg, A. B. (2017). The varieties of self-transcendent experience. Review of General Psychology, 21(2), 143-160.

Zeifman, R. J., \& Wagner, A. C. (2020). Exploring the case for research on incorporating psychedelics within interventions for borderline personality disorder. Journal of Contextual Behavioral Science, 15, 1-11.

Zinberg, N. E. (1984). Drug, set, and setting: The basis for controlled intoxicant use. Yale University Press. 